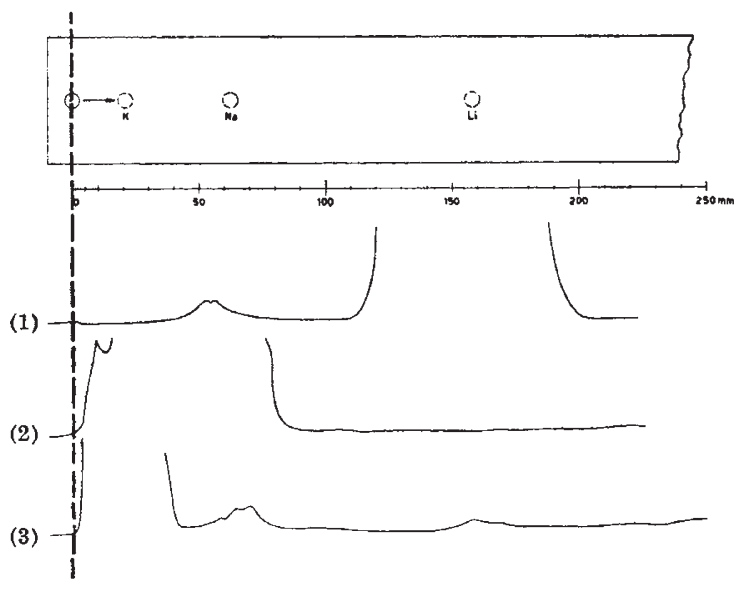

for potassium. $0.5 \mu \mathrm{gm}$. of lithium could be found even in presence of $500 \mu \mathrm{gm}$. of sodium.

Good separations were obtained also with the perchlorates, though the sensitivity of the method is then less satisfactory than with the chlorides.

Scheikundig Laboratorium

$$
\text { G. DE VRIES }
$$

der Vrije Universiteit,

Amsterdam.

Nov. 19.

1 Nature, 172, 542 (1953).

${ }^{2}$ Sakaguchi, T., and Yasuda, H., J. Pharm. Soc. Japan, 71, 1469 (1951); abstract in $Z$. anal. Chem., 137, 449 (1953).

\section{Photo- and Semi-conductance in Molecular Single Crystals}

THE following conclusions have arisen from work undertaken recently.

(1) Photo-conductance ${ }^{1}$ in molecular single crystals of the anthracene type is not an effect due primarily to impurity centres, since the dependence of photoconductance on wave-length reproduces all inflexions of the ultra-violet absorption curve of the crystal. Thus the photo-conductance of these molecular crystals is to be distinguished from that of many inorganic substances.

(2) Photo-conduction in anthracene, etc., should not be interpreted as a semi-conduction effect due to the conversion of the light into thermal energy, since, first, the amount of energy absorbed, which we have measured, is altogether inadequate, and, secondly, the current in the external circuit has been shown to depend on the number of quanta, rather than on the total energy absorbed. One electron enters the circuit for every $10^{4}$ quanta absorbed (approximately). Most adsorbed light is re-emitted ${ }^{2}$.

(3) To every region of conduction in the crystal there corresponds an electronic transition in the vapour. (A close relation between the crystal and the vapour spectra has been established ${ }^{3}$ in regions above $2000 \mathrm{~A}$. although such a relation is not true below 2000 A. ${ }^{4}$.) This conclusion applies with certainty to the singlet-singlet transitions and is probably also true for singlet-triplet transitions. In the latter case a simple interpretation of the results of other workers ${ }^{5,6}$ on semi-conduction becomes possible, namely, the energy derived from the temperature dependence of the conductance (anthracene ${ }^{5}, 13,300$ cm..$^{-1}$ ) is associated with the energy of excitation to the triplet-level (anthracene solution ${ }^{7}, 14,700 \mathrm{~cm}^{-1}$ ).

Such an association is supported by the following considerations : $(a)$ the population of the triplet-level becomes appreciable at the temperatures used; (b) the observed linear relation between $\log i$ and $1 / T$ is immediately accounted for, assuming that the current, $i$, is proportional to the number of electrons in the upper band; (c) the decrease in activation energy of semi-conductance with an increase in the number of aromatic rings is explained, since in general the energy of excitation to the triplet-level decreases with such an increase. This rule, which is being investigated theoretically, has exceptions, one of which is that the triplet-level of coronene lies at a higher energy than that of anthracene. However, the energy from semi-conductance of polycrystalline materials is greater for coronene than for anthracene in a very similar, though possibly fortuitous, proportion.

There is accordingly reason for believing that both photo- and semi-conduction are understandable in terms of the electronic transitions which are observed spectrally for these substances.

This work is being continued by two of us (D. J. C. and L.E. L.) and we shall publish a full theoretical and experimental account later. Dr. T. Iredale is thanked for his interest and encouragement, and the Vacuum Oil Co. for a scholarship (to D. J. C.).

D. J. Carswell

J. Ferguson

School of Chemistry,

University of Sydney. Dec. 18.

Vartanyan, A. T., Dok. Akad. Nauk S.S.S.R., 71, 641 (1951). Carswell, D. J., J. Chem. Phys., 21, 1890 (1953).

2 Bowen, E. J., Trans. Farad. Soc., 35, 56 (1939).

${ }^{3}$ Prikhotjko, A., J. Phys., U.S.S.R., 8, 257 (1944).

- Dunn, T., and Lyons, L. E. (unpublished work).

${ }^{5}$ Mette, H., and Pick, H., Z. Phys., 134, 566 (1953).

- Eley, D. D., Parfitt, G. D., Perry, M. J., fand Taysum, D. H., Trans. Farad. Soc., 49, 79 (1952).

: Lewis, G. N., and Kasha, M., J. Amer. Chem. Soc., 66, 2100 (1944).

\section{Fluorescent Spectra of Solid Solutions of Naphthalene with Added Anthracene}

Some time ago we investigated the fluorescent spectra of solid solutions of naphthalene with added naphthacene, naphthalene with added 1,2,5,6-dibenzanthracene, and anthracene with added naphthacene, under X-ray excitation 1 . We found that the fluorescence characteristic of the added compound increased with concentration to a maximum, and then fell off. The results for anthracene with added naphthacene agreed with those of Bowen ${ }^{2}$, and all the results could be explained on the basis of the exciton theory proposed by Bowen and his colleagues $^{2,3}$. The solid solutions were prepared by dissolving under gentle heat a small, carefully weighed amount of one compound in a known amount of the other. The samples were excited with X-rays, and the spectra were obtained by means of a Beckman Model DU quartz spectrophotometer used as a monochromator, with a 931-A. photomultiplier tube used as detector.

Some subsequent results obtained in the same manner from crystals of naphthalene with added anthracene are more complex; but a tentative explanation may be given with the aid of the exciton 